\section{Gradient Reference Specimens for Advanced Scanned Probe Microscopy ${ }^{\dagger}$}

\author{
Duangrut Julthongpiput, Michael J. Fasolka* \\ and Eric J. Amis
}

Polymers Division, Materials Science and Engineering Laboratory, National Institute of Standards and Technology, Gaithersburg, MD 20899 *mfasolka@nist.gov

Introduction: Scanned Probe Microscopy Methods and the Reference Specimen Paradigm

Scanned Probe Microscopy (SPM) techniques are attractive because they provide easily acquired micrographs that map specimen properties with nanometer scale resolution. SPM micrographs can be collected without the use of high vacuum (as opposed to many electron microscopies). Moreover, SPM methods allow in-situ imaging of specimens in a variety of environments, including under liquids and at higher temperatures. However, from a metrology perspective, the great promise of SPM must be balanced by the fact that SPM techniques generally provide qualitative data unless supplementary actions are taken. That is, quantification of SPM image contrast is difficult since it depends highly upon probe characteristics, probel sample interactions (both of which can be difficult to gauge), and instrument calibration.

For example, quantification of SPM topography data is hampered by two main factors. First, accurate interpretation of SPM image height contrast relies on precise calibration of the $z$-piezo element, which can suffer from non-linearity, voltage drift and hysteresis. Second, measured topography is necessarily a geometric convolution of the actual specimen topography and the probe shape and quality, ${ }^{1}$ which varies from probe to probe. Due to careful analysis and research, much of which was conducted at the National Institute of Standards and Technology (NIST), ${ }^{2}$ in principle these challenges to quantitative AFM topography have been met. Moreover, such research has resulted in commercially available reference specimens that allow individual users to conveniently calibrate height contrast and gauge probe shape in their own facilities.

SPM reference specimens are most useful when they link local instrument contrast to a well-established (i.e. traditional) measure-

Table 1: Short Survey of Next Generation SPM Techniques

\begin{tabular}{|l|l|l|l|}
\hline SPM TECHNIQUE & $\begin{array}{l}\text { Microscopy } \\
\text { Mechanism }\end{array}$ & $\begin{array}{l}\text { Proposed Factors that } \\
\text { Influence Contrast }\end{array}$ & $\begin{array}{l}\text { Quantification } \\
\text { Barriers }\end{array}$ \\
\hline $\begin{array}{l}\text { Chemical Force } \\
\text { Microscopy (CFM) }\end{array}$ & $\begin{array}{l}\text { Functionalized probe } \\
\text { enhances chemical } \\
\text { sensitivity to friction } \\
\text { and phase data }\end{array}$ & $\begin{array}{l}\text { A variety of local } \\
\text { chemical interactions, } \\
\text { environmental effects, } \\
\text { local mechanical } \\
\text { properties }\end{array}$ & $\begin{array}{l}\text { Quality of probe } \\
\text { functionalization, } \\
\text { environmental effects } \\
\text { (e.g. humidity), } \\
\text { combination effects, } \\
\text { topography artifacts }\end{array}$ \\
\hline $\begin{array}{l}\text { Atomic Force } \\
\text { Acoustic }\end{array}$ & $\begin{array}{l}\text { Cantileveriprobe } \\
\text { response to specimen } \\
\text { vibration frequency } \\
\text { sweep gives local } \\
\text { modulus }\end{array}$ & $\begin{array}{l}\text { Specimen modulus, } \\
\text { probe/specimen } \\
\text { adhesion, environmental } \\
\text { effects }\end{array}$ & $\begin{array}{l}\text { Environmental } \\
\text { effects, combination } \\
\text { effects (modulus/ } \\
\text { adhesion), unknown } \\
\text { measurement } \\
\text { volume }\end{array}$ \\
\hline $\begin{array}{l}\text { Friction Force } \\
\text { Microscopy (FFM) }\end{array}$ & $\begin{array}{l}\text { Torsional cantilever } \\
\text { deflection due to friction } \\
\text { force between probe } \\
\text { and specimen. }\end{array}$ & $\begin{array}{l}\text { Probe/specimen } \\
\text { adhesion, specimen } \\
\text { topography (roughness), } \\
\text { environmental effects }\end{array}$ & $\begin{array}{l}\text { Probe quality, } \\
\text { combination effects }\end{array}$ \\
\hline
\end{tabular}

ment. Regarding our current example, a now common route to height contrast ( $z$-piezo) calibration involves imaging of microfabricated reference gratings with a "known" amplitude. In many cases, such reference gratings are "traceable" through a NIST-developed SPM instrument specifically designed to relate piezo-derived height data to optical interferometery, which provides precise distance measurements. ${ }^{2}$ Reference specimens useful for gauging probe shape are also available. ${ }^{4 \ddagger}$ For example, specialized substrates with ultra sharp features, allow users to conveniently measure tip radius and quality by imaging the substrate. Tip shape data can then be used to "deconvolute" AFM micrographs, yielding more quantitative measurements of specimen topography. Indeed, computational procedures for performing this correction have been detailed by NIST researchers .

This illustrates the power of well-designed reference specimens for quantifying SPM topography data. In this article, we describe our research at NIST, which aims to extend the reference specimen paradigm to more complex SPM techniques.

\section{A New Research Program at NIST:}

Recent years have seen the emergence of a new generation of SPM techniques, which are designed to measure chemical, mechanical, and electro/optical properties on the nanoscale. Table 1 outlines a few of these methods. Currently, these techniques provide qualitative (or semi-quantitative) data, often expressed in terms of relative instrument parameters (e.g. piezo voltage or cantilever response). The development of quantitative SPM techniques require methods to gauge and accommodate the following factors:

- Resolution and Sensitivity: The lateral resolution, effective measurement volume, and sensitivity to the measured properties need to be determined for many new SPM techniques. Moreover, there is a need to calibrate these factors with respect to established analysis techniques such as spectroscopy.

- Topographic Artifacts: The "cartoon" scheme of advanced SPM is analysis of a planar specimen, yet topographic features are inherent to nearly all specimens. While it is well known that topography can muddy the measurement of other properties, there is currently no way to gauge or parse these effects in SPM.

- Environmental Effects: Ambient conditions can modify tipspecimen interactions, and, therefore, SPM contrast. A prime example is relative humidity, which affects the character of the water layer adsorbed onto specimen surfaces. The so called "meniscus force" exerted by this layer is an important factor in probe/sample interaction.

- Combination Effects: SPM measurements of, for example, mechanical properties can be convoluted with local adhesion, topography (see above) and other effects. It would be invaluable if there were a convenient means to gauge each of these effects separately.

- Probe quality: Many new SPM methods depend upon custom-made probes, the fabrication of which is often not reliable or uniform. In addition, such probes can wear during use. Accordingly, convenient means to characterize these probes (before and during use) are needed.

† Contribution of the National Institute of Standards and Technolog\%, not subject to copyright in the United States.

₹ Certain commercial materials are identified in this paper to foster understanding. Such identification does not imply recommendation or endorsement by the National Institute of Standards and Technology, nor does it imply that the materials identified are necessarily the best available for the purpose. 


\section{thinkforward}

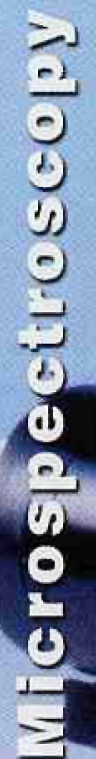

Tie Teerer fiti

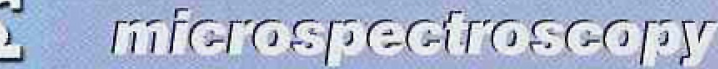

b) Bruker Optics offers the

d) most advanged FTF,

- FTrFaman and Dispersive

Reman microspectroscopy

tools to meet all your

demanding microanalysis reguirrements. The countless fimovations found on our microspectrometers, epitomize oui pliflosophy of think forward

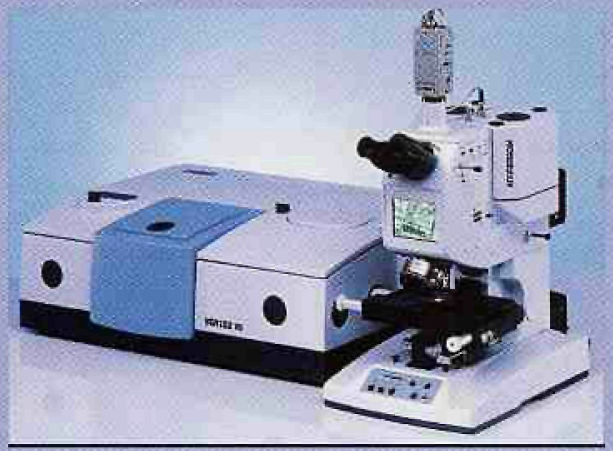

\section{HYPERION ${ }^{\text {TM }}$ Series}

The HYPERION ${ }^{\mathrm{m}}$ Series infrared microscope offers state-of-the-art optical microscopy capabilities, which include contrast enhancement with aperture stops, visible polarization, darkfield illumination and fluorescence illumination. The HYPERION ${ }^{\text {TM }}$ Series also offers unmatched infrared performance, designed to maximize infrared throughput without sacrificing diffraction limited performance. This translates to shorter collection times with the highest quality data possible. The HYPERION ${ }^{\text {TM }}$ is a fully upgradeable microscope platform, from a basic high performance infrared microscope with transmission and reflection capabilities to a dual detector focal-plane-array and single element detector based system.

\section{The HYPERION ${ }^{T M}$ Series Advantage}

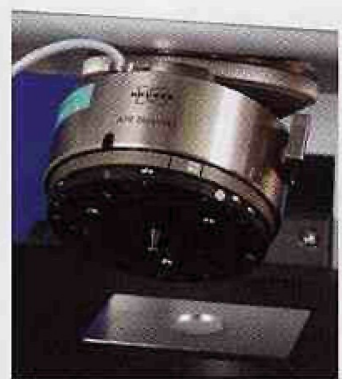

\section{Proprietary ATR}

Objective

- Crystal-clear sample viewing

- Unmatched infrared performance

a 5 pressure settings with automatic pressure detection

$\square$ Anvil ATR crystal design with 80 micron contact area

- ATR mapping

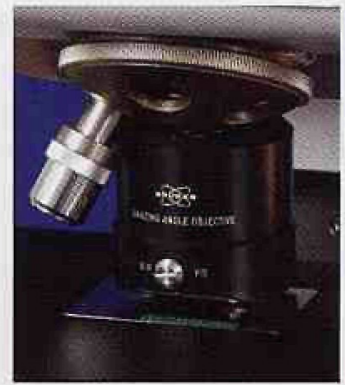

\section{Patented Grazing}

Incidence Objective

- Crystal-clear sample viewing

- ALL infrared light rays used at the correct angles of incidence a 2 reflections off sample

a Breakthrough sensitivity in analyzing thin films and monolayers

- Grazing incidence analysis of monolayers to $20 \times 20$ microns

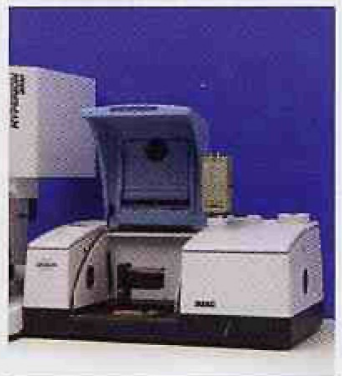

\section{Focal Plane Array} Micro/Macro Analysis

- Upgradeable from base HYPERION 1000

a Portable FPA can be easily moved between HYPERION ${ }^{\prime \prime}$ microscope and macrochamber

- "Next generation" FPA detectors utilized

- Lower cost

For more information:

please call 1-888-4BRUKER or visit www.brukeroptics.com/microscopy

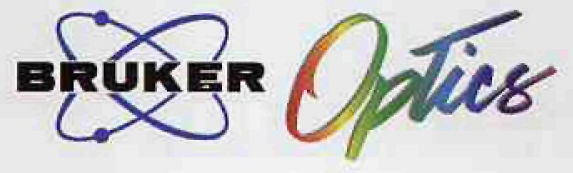


Of course, quantification of these factors will result in a better understanding of probe/specimen interactions - the ultimate scientific goal of SPM metrology.

In response to these needs, a research program recently initiated by the NIST Combinatorial Methods Center (NCMC) aims to provide a suite of reference specimens useful for the quantification of next-generation SPM data. By design, these specimens will characterize SPM probes, calibrate SPM image contrast with "traditional" surface measurements (e.g. spectroscopy, contact angle) and provide information useful for modeling complex probe/sample interactions. This is a demanding set of objectives, which are attainable because our reference specimens leverage design principles and fabrication techniques developed for combinatorial research. Combinatorial specimen libraries express a multitude of properties on a single substrate. So, while a traditional reference substrate gives a single point calibration, a combinatorial specimen can calibrate SPM contrast over a large range of properties. Moreover, combinatorial specimens can vary two or more properties in a systematic and independent manner. Accordingly, such specimens provide means to parse "combination factors" discussed above.

The NCMC has developed a collection of "gradient" combinatorial methods, ${ }^{5}$ used to fabricate specimens that continuously vary in properties such as surface energy (chemisty/adhesion), film thickness, composition, topography (roughness) and modulus - qualities which are known to govern SPM contrast. By combining these gradient tools with bench-top microfabrication techniques, specimens with a graded property pattern are created. These micron-scale patterns are convenient for SPM imaging, and (as discussed below) allow image contrast to be gauged against a constant background.

\section{An Example Gradient Reference Specimen Design and Its Fabrication:}

Figure 1 schematically illustrates an example reference specimen. We are currently testing this specimen design as a means to quantify chemically sensitive SPM techniques such as friction-force AFM, or Chemical Force Microscopy (CFM), which employs a chemically functionalized AFM probe. ${ }^{6}$ The central part of the reference specimen has a pattern of micron-scale lines that gradually change in their chemisty (e.g. surface energy) with respect to a constant matrix. Imaging along the patterned region with SPM, provides a series of micrographs with systematically changing contrast. Wide, chemically smooth "calibration" fields, which bound the patterned area, directly reflect the changing chemistry of the lines and the static chemistry of the matrix, such that traditional (e.g. contact angle) measurements along the calibration fields gauge chemical differences in the gradient micro-pattern. Accordingly, for SPM micrographs acquired along

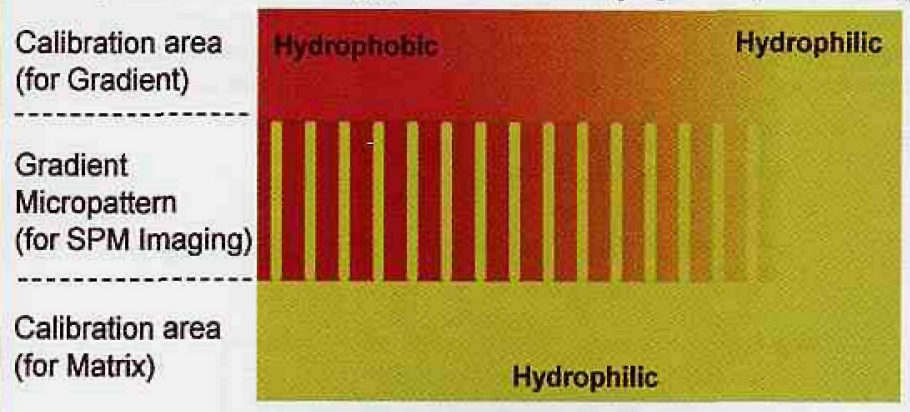

Figure 1: Schematic of combinatorial gradient reference specimen for gauging tip quality, for calibrating image contrast, and for determining sensitivity in chemically sensitive SPM techniques. Image contrast in the patterned area is gaiged via traditional measurements along the calibration areas.

\section{Print with SAM A}

\section{Fill with SAM B}

${ }^{*}$ Composite Elastomer Stamp*

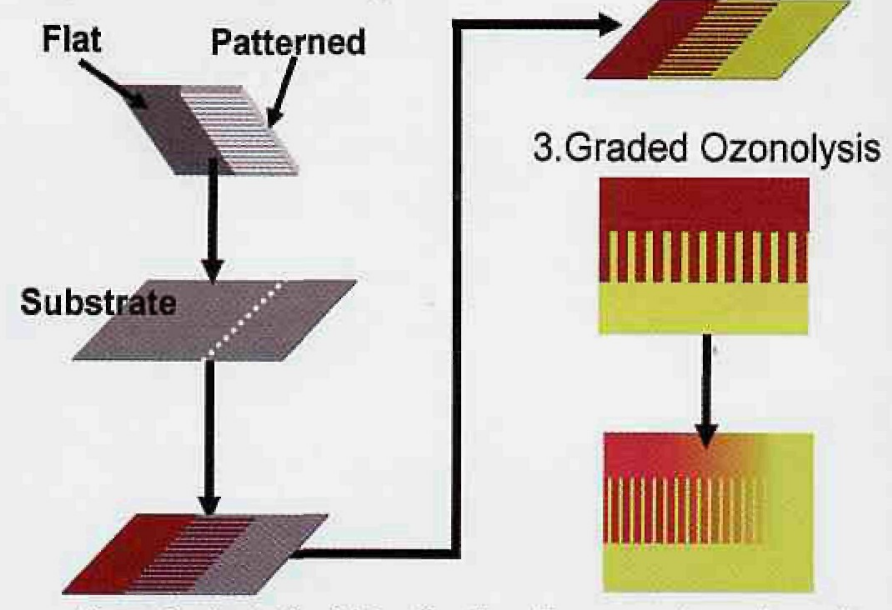

Figure 2: Route for fabricating the reference specimen shown in Figure 1. SAMA (hydrophobic) is printed with a composite elastomer stamp to form micron scale lines and an adjacent calibration field. Emersion in a solution or vapor of SAM B (hydrophilic) forms the matrix (with adjacent calibration field). A graded UV-ozonolysis of $S A M A$ gradually converts it into species similar to $S A M B$, resulting in a complete specimen

the patterned region, the specimen calibrates the image contrast with respect to the traditional measurements. In addition, the specimen illuminates the sensitivity of the probe, which is related to the position along the gradient where image contrast is first observed. Similarly, for techniques like CFM, this specimen serves as a tool for comparing the quality of tip-functionalization.

As shown in Figure 2, fabrication of this gradient-pattern specimen requires soft-lithography of appropriate SAM molecules onto a planar substrate. A composite elastomer stamp, which has both flat and corrugated areas, allows printing of the micropatterned strip with the adjacent solid calibration field. Next, a graded UV-ozonolysis (UVO), gradually modifies the chemistry of the patterned SAM (and calibration field) along one direction. ${ }^{5,7}$ For example, methylterminated alkyl chain monolayers (hydrophobic) can be gradually converted into carboxylic acid terminated (hydrophilic) chains ${ }^{7}$ - as one travels down the specimen, the density of hydrophobic molecules decreases, while hydrophobic chains increase. Subsequent "filling" with a hydrophilic SAM completes the "matrix" of the specimen.

We tested this design and process by micro-contact printing hydrophobic thiol-SAM "ink" onto gold substrates followed by gradient UVO exposure and filling with a hydrophilic thiol layer. Figure 3 shows data collected from this prototype. The central graph shows water contact angle measurements taken on the calibration strips. Here, the matrix measurements (hydrophilic SAM) remain constant along the specimen, while the contact angle decreases along the graded calibration strip as it changes from an hydrophobic to hydrophilic SAM. A series of friction force AFM measurements acquired along the gradient micropattern demonstrates how the friction contrast decreases as the chemical nature of the lines and matrix become increasingly similar. By correlating the position of the micrographs with the calibration data, a calibration of image contrast with respect to surface energy differences is possible.

\section{Future Research Directions:}

While our prototype validates the specimen design, ultimately the use of delicate gold/thiol chemistry is not very useful for refer- 


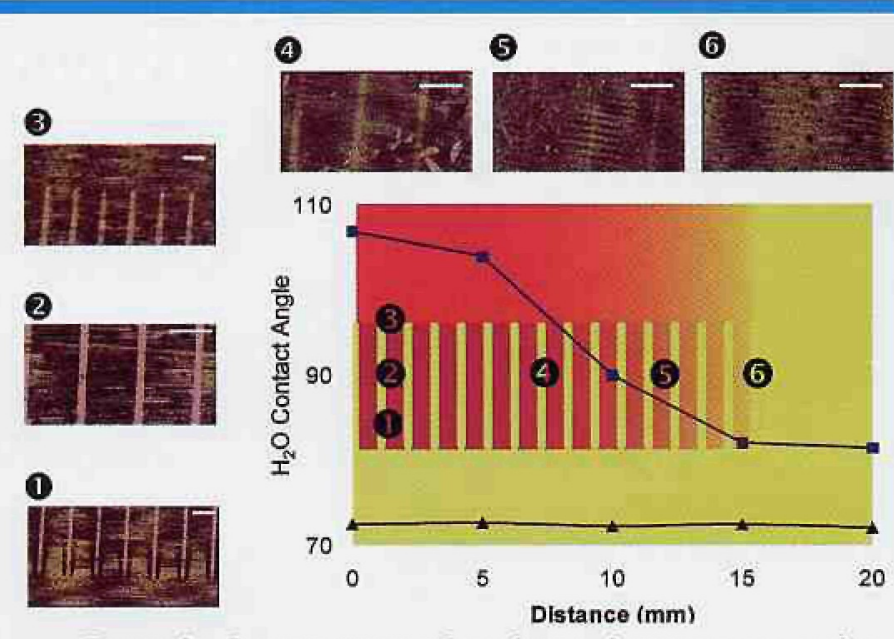

Figure 3: Demonstration of gradient reference specimen for chemically sensitive SPM techniques. This specimen reflects the design in Figure 1 and was fabricated as in Figure 2 using thiol SAMs on a gold substrate. The central graph shows contact angle measurements along the calibration fields (uncertainty $\approx 10 \%$ ). The blue squares show the gradient calibration, black triangles show the matrix calibration. Surrounding friction force AFM measurements (constant z-scale: 0.2 $\mathrm{mV})$ show diminishing image contrast aiong the micropatterned region $(0,0,5,0)$ and validate the printing integrity $(0,3)$. White scale bars are $10 \mathrm{um}$.

ence substrates, since the patterns degrade over a few days. We have successfully fabricated specimen prototypes using more robust substrates and chemistries (monochlorosilane SAMs on $\mathrm{SiO}_{2}$-terminated silicon). Ultimately, however, we aim to create combinatorial micropattern specimens using a compositionally graded layer of grafted polymers. These substrates will last indefinitely through use and cleaning cycles; making them viable as NIST reference materials. Currently, we are testing the gradient chemical micropatterns discussed in this article for the calibration of emerging SPM techniques such as CFM and Atomic Force Acoustic Microscopy. ${ }^{8}$ In addition, we are developing new designs that exhibit patterned gradients in topography, modulus, and adhesion, which can be used to calibrate a variety of other SPM methods and nanomechanical testing techniques such as nanoindentation.

For more information on the NCMC, this research program, or to provide feedback on this effort, please contact us at combi@nist. gov or visit the NCMC website at http://www.nist.gov/combi.

\section{Acknowledgements:}

The authors thank the NIST MSEL Director for Funding. Thanks also to Donna Hurley (Materials Reliability Division, NIST), Tinh Nguyen, Xiohong Gu (Building and Fire Research Laboratory, NIST) and Sergei Magonov (Veeco) for providing feedback on our specimen designs.

\section{References:}

I. J.S. Villarrubia, J. Res. Nati. Inst. Stand. Technol., 102, 425 (1997).

2. R. Dixson, R. Koning, V. Tsai, J. Fu, and T. Vorburger, Proceedings of the SPIE 3677 (1-2), 20 (1999).

3. See http://s.nist.gov/traceability, for more information on NIST Traceable Standards

4. See, for example, http://www:spmtips.com/products/gratings/

5. A. Sehgal, A. Karim, C.F. Stafford, and M.J. Fasolka, Microscopy Today 11, 26 (2003)

6. A. Noy, D.V. Vezenov, and C.M. Lieber, Annu. Rev. Mater. Sci, 27, 381 (1997).

7. S. V. Roberson, A. J. Fahey, A. Sehgal, and A. Karim, Applied Surface Science, 200,150 (2002).

8. See e.g., D. C. Hurley and J. A. Turner, J. Appl. Phys., 95, 2403 (2004).

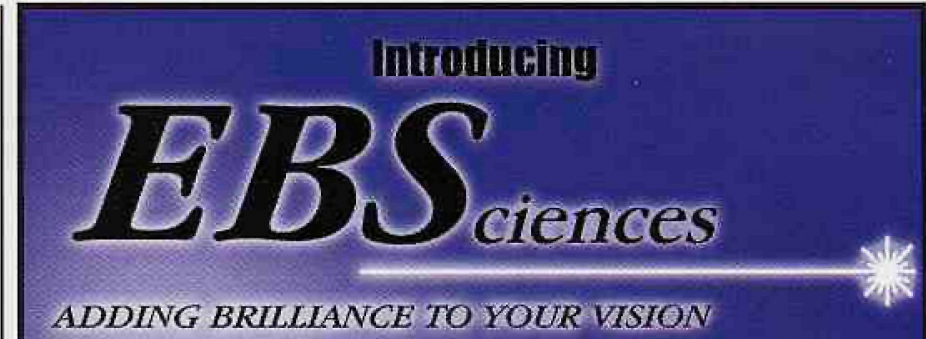

as the Exclusive U.S. Distributor for:

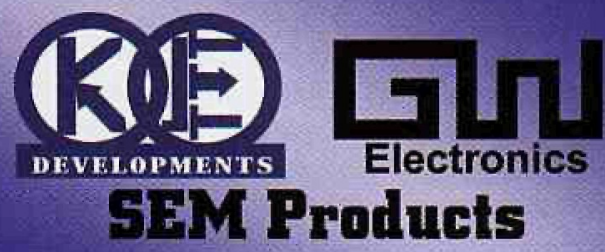

Chamber Surveillance Systems

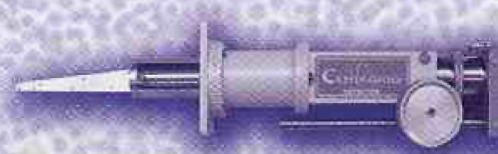

Detectors
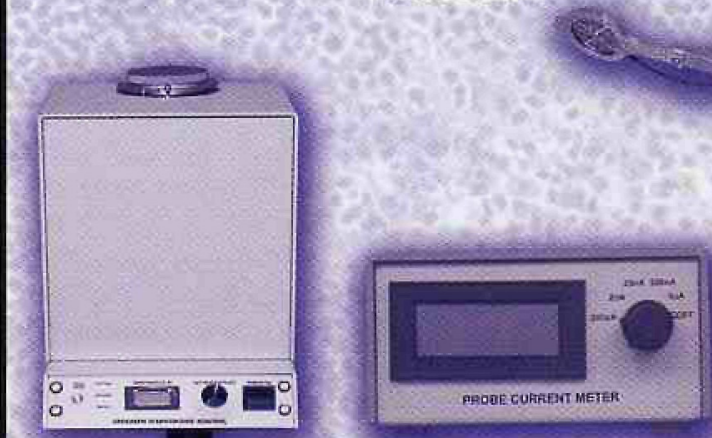

Accessories

Introiluctory/him Special $25 \%$ OFF Infrarei Chamberseone

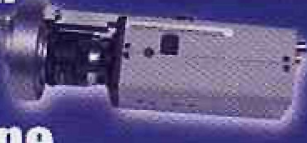

Energy Beam Sciences Inc. 800-992-9037 or 413-786-9322 email: ebs@ebsciences.com www.ebsciences.com

See us at M\&M in Savannah, GA. Booth \#721 